\title{
Téoros
}

Revue de recherche en tourisme

Janice Deledalle-Rhodes. L'Orient représenté. Charles Montagu

Doughty et les voyageurs anglais $d u X^{X} X^{e}$ siècle. New York:

Peter Lang, 2000

\section{Martine Geronimi}

Volume 21, numéro 1, printemps 2002

URI : https://id.erudit.org/iderudit/1071541ar

DOI : https://doi.org/10.7202/1071541ar

Aller au sommaire du numéro

Éditeur(s)

Université du Québec à Montréal

ISSN

0712-8657 (imprimé)

1923-2705 (numérique)

Découvrir la revue

Citer ce compte rendu

Geronimi, M. (2002). Compte rendu de [Janice Deledalle-Rhodes. L'Orient représenté. Charles Montagu Doughty et les voyageurs anglais du XIX ${ }^{e}$ siècle. New York : Peter Lang, 2000]. Téoros, 21(1), 62-63.

https://doi.org/10.7202/1071541ar

Ce document est protégé par la loi sur le droit d'auteur. L'utilisation des services d'Érudit (y compris la reproduction) est assujettie à sa politique d'utilisation que vous pouvez consulter en ligne.

https://apropos.erudit.org/fr/usagers/politique-dutilisation/
Cet article est diffusé et préservé par Érudit.

Érudit est un consortium interuniversitaire sans but lucratif composé de l’Université de Montréal, l'Université Laval et l'Université du Québec à Montréal. Il a pour mission la promotion et la valorisation de la recherche. https://www.erudit.org/fr/ 


\title{
Janice Deledalle-Rhodes. L'Orient représenté. Charles Montagu Doughty et les voyageurs anglais du XIX' siècle.
}

\author{
New York: Peter Lang, 2000.
}

\section{Martine Geronimi}

Dans ce livre, L'Orient représenté, Janice Deledalle-Rhodes aborde la question clé de lá représentation de l'Autre à travers le voyage. Remarquons que l'Orient était une destination fort prisée par les Européens durant tout le XIX siècle.

L'auteure de ce livre pénétrant s'intéresse à la vision anglaise des lieux et de leurs habitants. Il paraît capital de noter que les récits de ces voyageurs fournissent une contre-lecture, celle de la vision des Orientaux de leurs visiteurs anglais. Du moins, par un jeu de contre-miroir, les Oceidentaux témoignent implicitement de la perception des Orientaux, représentation forgéc à leur contact réciproque.

Arabia Deserta, relation du voyage de Charles Montagu Doughty, se veut le modèle de référence de la littérature des voyageurs anglais du XIX ${ }^{\circ}$ siècle, selon l'auteure. Arabia Deserta dépeint la prise de conscience affective de l'Étre Oriental par le voyageur anglais. C'est pourquoi ce récit apparaît effectivement comme le témoignage d'une expérience totale dans un monde d'émotions et de tensions, "un monde où l'on ne retrouve aucune trace d'exotisme superficiel de l'époque $*$ (p.68).

Mais quel est cet Orient dépeint dans tous ces récits ? Il correspond en fait au « Moyen-Orient $»$ contemporain, à une aire géographique a où s'exerce l'influence de l'Islam et de la civilisation islamique, plus forte que le Désert, plus forte que les Etats, plus forle que les gégraphes o (p. 14).

La période choisie dans cette étude est fixée très précisément, de 1809 à 1896 . L'année 1809 marque le voyage à Constantinople de Lord Byron et de J.C. Hobbhouse, moment fertile où Hobbhouse prenait conscience de l'existence effective de l'Oriental qui lui restait inaccessible. La date finale révèle définitivement un changement de mentalité chez les visiteurs anglais, en l'occurrence W.B. Harris, pour qui l'Oriental est présenté comme l'Égal de I'Anglais.

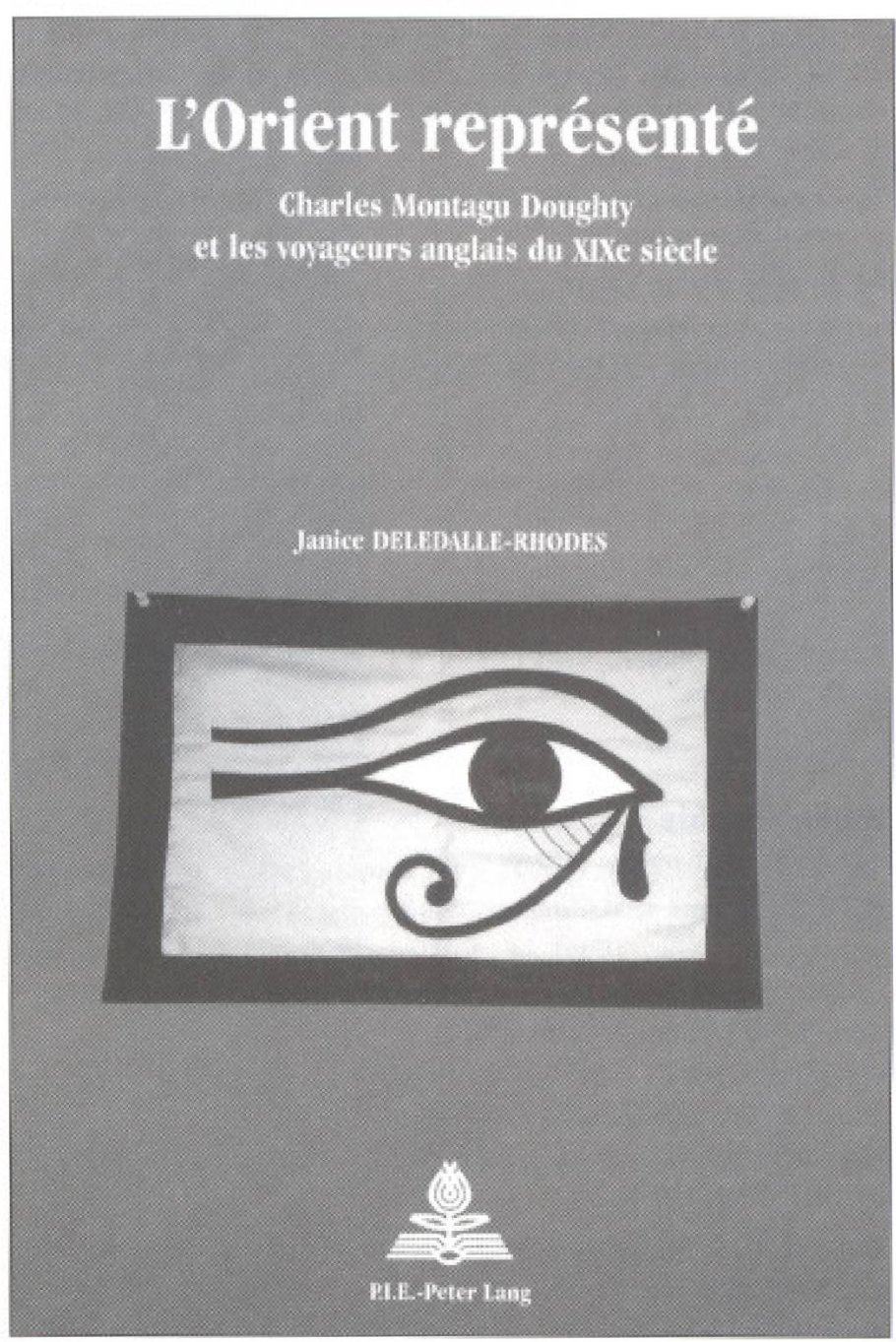

Les motivations principales des voyageurs, relevées parl l'auteure, sont conformes à l'idée que l'on a de ce siècle colonisateur. La mission d'information est la première, suivie de la mission * civilisatrice $\%$. Ainsi, les Anglais sont persuadés d'apporter le progrès, à la fois matériel et politique. A ces motivations s' interpénètrent, tout au long du siècle, des intentions très personnelles 
donnant lieu à des formes de tourisme culturel et exotique : un goût pour le tourisme d'évasion.

L'auteure, à la lecture des différentes relations, a su dégager, en les catégorisant, des stéré́otypes positifs et négatif́s concernant les Orientaux, mais aussi les Occidentaux vus par les Orientaux. Cette source se montre fort précieuse en nos temps troublés par les questions du Proche-Orient. Une riche collection de regards croisés mérite toute notre attention (deuxième partie du livre p. 123-243). Les clichés négatifs abondent de part et d'autre. L'Oriental barbare et décadent se double d'un menteur et d'un hypocrite, tandis que l'Occidental est vu comme un espion à la fois sorcier et magicien ou chercheur de trésors. Ces visions sont tempérées par d'autres, plus favorables et toutes aussi classiques que la légendaire hospitalité des Orientaux ou le raffinement de leur mode de vie. Ce que montre Janice DeledalleRhodes, c'est une transformation de l'attitude des voyageurs au contact de l'Autre different. Quatre attitudes affleurent chez les voyageurs anglais : au départ l'Oriental est vu comme a un instrument de voyage $*$ (p. 241) ; plus tard, il devient l'objet d'une étude, \& un spécimen curieux $\%$. Dans un troisième moment, la réalité humaine de l'Oriental apparât au voyageur qui est alors obligé de se confronter sur le plan de l'égalité. Enfin, plus rarement mais effectivement, le woyageur s'identifie totalement à l'Oriental.

Chez les Orientaux, les attitudes négatives envers l'Anglais inspirant haine, mépris, voire répugnance, se transforment en conduite d'acceptation si l'Occidental montre un comportement d'ouverture et d'adaptation à la situation culturelle nouvelle. Quand la relation dépasse les blocages sociaux et que les rapports interpersonnels s'établissent, il y a alors place pour l'amitié.

Cependant, $1^{\prime}$ Oecidental se comporte de manière abstraite, voire ignorante des enjeux qui le concernent. L'aventure orientale est un projet raisonné, intellectualisé, celui d'un homme de science qui vient observer, alors que l'Oriental visité reçoit le voyageur sans l'avoir invité. Il est dans une attitude passive. II subit le voyageur. Il l'accueille avec «ses émotions» mêlant la peur, la surprise, le dégoût ou la détestation. Il reste cependant fidèle à son devoir d'hospitalité et accueille l'Autre le mieux possible. Mais, le voyageur anglais reste toujours, l' Infidele, le Kafir, celui que l'Arabe traite avec politesse mais distance. Le Musulman se sent supérieur à cet Occidental parce qu'il a la foi en l'Islam qui lui apparaît comme une religion parfaite et unique, seule capable de l'amener à dominer le monde. En effet, le voyageur décrit le Musulman comme un être convaincu de pouvoir imposer ses conceptions religieuses «quelque puisse être, par ailleurs, la supériorité de son interlocuteur occidental» (p. 272).

L'Anglais perçoit l'Orient, selon sạ vision d'homme de progrès, à partir du débat constant du XIX ${ }^{2 m e}$ siècle opposant science et religion. Janice Deledalle-Rhodes identifie chez les voyageurs anglais quatre attitudes différentes qui s'entremêlent et complexifient la vision de l'Ailleurs. Soit, ils critiquent ou défendent des aspeets spécifiques de l'Islam, soit, ils analysent la religion musulmane comme ils le feraient pour d'autres religions et en particulier la leur. L'attitude de Charles Montagu Doughty est révélatrice de la complexité de ce regard porté sur la religion musulmane. Farouchement protestant, Doughty est cependant un homme de science à la recherche de la vérité. Le premier reproche fait à l'Islam est son fatalisme que Doughty considère comme ennemi du progrès. Si Arabiata Deserta est un ouvrage scientifique, il n'en demeure pas moins une expé rience intime, où le vécu teinté de subjectivité prend le pas sur l'attitude objective et froide du chercheur. Malgré son intransigeance religieuse, le voyageur relève, chez les Musulmans, des caractéristiques coïncidant avec sa vision protestante pétrie d'austêrité et de sobriété. Faits êt impressions s'entrecroisent et tissent une toile de paradoxes dans le récit de ce voyageur.

L'auteure souligne le fait que tous ces Anglais, qui se déplacent dans ce Moyen-Orient, sont à la recherche d'une religion naturelle quasi instinctive. Elle démontre que ces voyageurs méconnaissent l'Islam et que, sur place, ils n'y retrouvent «que le reflet des questions qui préoccupent l'Angleterre à cette époque $\$(\mathrm{p}$ - 322). De plus, cette quête de l'Autre n'est pas neutre chez les Anglais. Elle s'accompagne de prises de positions politiques. L'auteure en dévoile trois principales. La première reprend ce besoin intéressé d'une grande puissance à établir sa suprématie stratégique sur le Moyen Orient. L'Angleterre est alors une puissance hégémonique ; la seconde repose sur la mission civilisatrice chère au XIX ${ }^{\text {tmo }}$ siècle colonisateur. La Grande Bretagne se doit d'apporter le Progrès aux Musulmans ; la dernière repose sur l'opposition philosophique absolue à tout régime politique despotique. L'Angleterre doit apporter un régime politique aux valeurs démocratiques. Ces trois raisons nous semblent contenir les germes de la situation malheureuse qui secoue la Palestine et le monde.

En essayant de prendre en compte le point de vue de l'Islam, l'auteure fait montre d'une sensibilité remarquable. $\mathrm{A}$ la fois instructif, intelligent et bien documenté ce livre mérite d'être médité en cette période troublée.

Martine Geronimi est stagiaire postdoctorale à la Chaire de recherche du Canada sur le patrimoine urbain et au Centre interuniversitaire d'études sur les lettres, les arts et les traditions à l'Universiné du Québec à Montréal. Elle est aussi adjointe à la rédaction de Téoros. 